\title{
Laser scanned photodiodes (LSPs) for image sensing
}

\author{
M. Vieira ${ }^{\mathrm{a}, *}$, M. Fernandes ${ }^{\mathrm{a}}$, Yu. Vygranenko ${ }^{\mathrm{a}}$, P. Louro ${ }^{\mathrm{a}}$, R. Schwarz ${ }^{\mathrm{a}}$, M. Schubert ${ }^{\mathrm{b}}$ \\ ${ }^{a}$ Electronics and Communications Department, ISEL, R. Conselheiro Emídio Navarro, P 1949-014 Lisboa, Portugal \\ ${ }^{\mathrm{b}}$ Institut fur Physikalische Elektronik, Universitat Stuttgart, Pfaffenwaldring 47, D-70569 Stuttgard, Germany
}

Received 11 June 2001; received in revised form 25 October 2001; accepted 8 November 2001

\begin{abstract}
An optimized $\mathrm{ZnO}: \mathrm{Al} / \mathrm{a}-\mathrm{pin} \mathrm{Si}_{x} \mathrm{C}_{1-x}: \mathrm{H} / \mathrm{Al}$ configuration for the laser scanned photodiode (LSP) imaging detector is proposed and the readout parameters improved. The effect of the sensing element structure, cell configuration and light source flux are investigated and correlated with the sensor output characteristics. Data reveals that for sensors with wide band gap doped layers an increase on the image signal optimized to the blue is achieved with a dynamic range of two orders of magnitude, a responsivity of $6 \mathrm{~mA} \mathrm{~W}^{-1}$ and a sensitivity of $17 \mu \mathrm{W} \mathrm{cm}^{-2}$ at $530 \mathrm{~nm}$. The main output characteristics such as image responsivity, resolution, linearity and dynamic range were analyzed under reverse, forward and short circuit modes. The results show that the sensor performance can be optimized in short circuit mode. A trade-off between the scan time and the required resolution is needed since the spot size limits the resolution due to the cross-talk between dark and illuminated regions leading to blurring effects. (C) 2002 Elsevier Science B.V. All rights reserved.
\end{abstract}

Keywords: Laser scanned photodiode (LSP); Image acquisition and representation; Output characteristics; Read-out parameters

\section{Introduction}

Image sensors used for commercial applications [1-5] are based on arrays of sensing elements and use essentially the same light sensing mechanism. Photons penetrating a depletion region generate electron-hole pairs. These carriers are swept away by the electric field across the depletion region and generate a small transverse photocurrent. In the past it was proposed a new kind of image sensor, the laser scanned photodiode (LSP) [6]. This sensor is fundamentally different from the other electrically scanned image systems. It consists on one large area $\mathrm{p}-\mathrm{i}-\mathrm{n}$ sensing element and an optomechanical acquisition system. The image to be acquired is optically mapped on the photosensitive surface using a conventional imaging system and a low-power chopped laser scans the sensor in the raster mode. The LSP sensor could present some advantages when compared with the conventional CCD, such as no special photolithography techniques or elaborated processing algorithms are needed. Also the use of the amorphous silicon technology allows the production of large area sensors increasing the image area without the need of additional optics.

The physical process that supports the laser scanning image acquisition is explained by the modulation of the

\footnotetext{
${ }^{*}$ Corresponding author. Tel.: +351-21-8317181; fax: +351-21-8317114.

E-mail address: mv@isel.pt (M. Vieira).
}

electrical field across the junction under a steady-state light pattern [7]. Low electric fields are ascribed to illuminated regions and high electric fields to dark zones [8]. When a weak light spot scans the device, in the dark regions the carriers generated by the probe beam are separated by the electric field and collected while those generated inside the illuminated regions mostly recombine inside the amorphous bulk and are not collected.

Several enhancements on the device structure were proposed $[6,8,9]$ to optimize sensor performance. Further optimization can be achieved by a correct choice of the read-out parameters, like scanner beam spot size and sensor electrical bias. This work aims to clarify the possible improvements, physical limits and performance of LSP sensor. The read-out parameters are optimized and the effects of the sensor structure on the output characteristics are discussed.

\section{Theory of operation and image representation}

The LSP is fundamentally different from the other electrically scanned image systems since it is based on one single sensing element, and uses a modulated low-power beam of laser light to scan the active area directly. The LSP sensor is a two-dimensional structure composed of a $\mathrm{p}-\mathrm{i}-\mathrm{n}$ photodiode (the active element), transparent front and metal back contacts. The physical structure under a light pattern illumination is displayed in Fig. 1. 


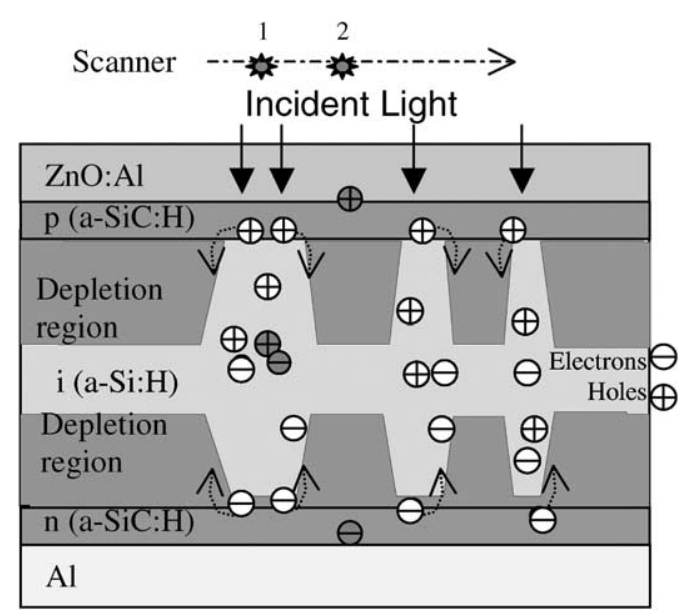

Fig. 1. Configuration and charge movement in a $\mathrm{p}-\mathrm{i}-\mathrm{n}$ structure under a light pattern illumination. The depletion region modulation and the carrier movement are superimposed.

A focused image, constituted by a steady-state light pattern, is projected onto the photosensitive surface through the transparent contact. For image acquisition, a low-power chopped laser scans the sensor in the raster mode. The readout of the injected carriers is achieved by measuring the ac component of the short circuit current, $i_{\mathrm{ac}}$, which depends on the intensities of both the image and the scanner. This component can be analytically described by the equation:

$i_{\mathrm{ac}}=R\left(\Phi_{\mathrm{L}}\right) \Phi_{S} \frac{\pi d^{2}}{4}$

where $R\left(\Phi_{\mathrm{L}}\right)$ is the small signal responsivity at a given illumination $\Phi_{\mathrm{L}}, \Phi_{S}$ is the average power density of the scanner beam and $d$ the beam diameter.

The signal-to-noise power ratio $(\mathrm{S} / \mathrm{N})$ depends on both components of the photocurrent $\left(i_{\mathrm{ac}}\right.$ and $\left.I_{\mathrm{dc}}\right)$ and is given by [10]:

$\frac{S}{N}=\frac{i_{\mathrm{ac}}^{2}}{\left(4 k T / R_{0}+2 q I_{\mathrm{dc}}\right) \Delta f}$

where $\Delta f$ is the bandwidth and $R_{0}$ the sensor resistance at zero voltage bias.

An optical system enables the change of the image and scanner beam intensity. All the measurements are performed with a chopper frequency $<1 \mathrm{kHz}$ and a scanner wavelength of $633 \mathrm{~nm}$. The $i_{\mathrm{ac}}$ component is measured using a lock-in amplifier. The scanning and acquisition process are controlled by a microcomputer which stores the currents as a two-dimensional array of discrete values, $i_{m, n}$, each one representing the photocurrent induced by the chopped light at the selected position. An $m \times n$ grid is used for image representation. The image intensity, $I_{m, n}$, is obtained by subtracting the background (without image, $i_{\mathrm{ac}}(0)$ ) from the input matrix $i_{m, n}$ (with image, $i_{\mathrm{ac}}\left(\Phi_{\mathrm{L}}\right)$ ).

The laser scanning image acquisition process is explained by the junction electrical field modulation under non-uniform steady-state illumination [7]. In a SiC:H/a-Si:H/SiC:H $\mathrm{p}-\mathrm{i}-\mathrm{n}$ structure the band misalignment is responsible by an increased ratio between the electrical field strength in dark and under illumination inside the i-layer [11]. At the illuminated regions the potential drop across the a-SiC:H doped layers increases leading to an inversion of electric field in the bulk while at the dark regions it remains high and the potential drop across the doped layers remains insignificant. If, in addition, a weak light spot is scanning the device, in the dark regions (position 2) the carriers generated by the probe beam are separated by the junction electric field and collected (high $i_{\mathrm{ac}}$ value). Those generated inside the illuminated regions (position 1) can diffuse or drift in the lateral direction. Nevertheless, a large number of them will recombine inside the amorphous bulk (low $i_{\mathrm{ac}}$ value). So, illuminated regions are ascribed to low ac components of the photocurrent and dark regions to high ac values.

\section{LSP deposition and characterization}

Large area $\mathrm{p}-\mathrm{i}-\mathrm{n}$ image transducers $(4 \mathrm{~cm} \times 4 \mathrm{~cm})$ in a glass/ZnO:Al/p $\left(\mathrm{Si}_{x} \mathrm{C}_{1-x}: \mathrm{H}\right) / \mathrm{i}(\mathrm{Si}: \mathrm{H}) / \mathrm{n}\left(\mathrm{Si}_{x} \mathrm{C}_{1-x}: \mathrm{H}\right) / \mathrm{Al}$ configuration were produced by PECVD [12]. The deposition conditions where kept constant for all i-layers, while they varied in the doped layers by adding methane during the deposition process. All the layers on sample \#M006291 are based in amorphous hydrogenated silicon (a-Si:H), while the p-layer in \#M006301 and the p- and n-layers in \#M007192 sensors are based on a-SiC:H alloy.

A preliminary electrical and optical characterization of the films was carried out by measuring the electrical conductivity in the coplanar direction and by obtaining the absorption spectra from transmission and reflection measurements. All the structures were characterized by spectral response, current-voltage and capacitance-voltage measurement in dark and under different optical bias conditions as described elsewhere [11]. The dark conductivity, $\sigma_{\mathrm{d}}$, the activation energy, $\Delta E$, the optical Tauc gap, $E_{\mathrm{op}}$, and the photoconductivity, $\sigma_{\mathrm{ph}}$, under AM1.5 $\left(100 \mathrm{~mW} \mathrm{~cm}^{-2}\right)$, for the analyzed sensors are summarized in Table 1 .

Table 1

Optoelectronic properties of the individual layers

\begin{tabular}{llllll}
\hline Cell code & Layers & $\begin{array}{l}\sigma_{\mathrm{d}} \\
\left(\Omega^{-1} \mathrm{~cm}^{-1}\right)\end{array}$ & $\begin{array}{l}\Delta E \\
(\mathrm{eV})\end{array}$ & $\begin{array}{l}E_{\mathrm{op}} \\
(\mathrm{eV})\end{array}$ & $\sigma_{\mathrm{ph}} / \sigma_{\mathrm{d}}$ \\
\hline \#M006291 & $\mathrm{p}$ & $8.2 \times 10^{-7}$ & 0.499 & 1.80 & 7.3 \\
& $\mathrm{i}$ & $7.6 \times 10^{-11}$ & 0.739 & 1.79 & $7.1 \times 10^{4}$ \\
& $\mathrm{n}$ & $7.8 \times 10^{-7}$ & 0.426 & 1.82 & 1.2 \\
\#M006301 & $\mathrm{p}$ & $8.2 \times 10^{-7}$ & 0.499 & 1.80 & 7.3 \\
& $\mathrm{i}$ & $7.6 \times 10^{-11}$ & 0.739 & 1.79 & $7.1 \times 10^{4}$ \\
& $\mathrm{n}$ & $1.9 \times 10^{-12}$ & 0.834 & 2.10 & 21 \\
\#M007192 & $\mathrm{p}$ & $2.5 \times 10^{9}$ & 0.649 & 2.06 & 4.5 \\
& $\mathrm{i}$ & $7.6 \times 10^{11}$ & 0.739 & 1.79 & $7.1 \times 10^{4}$ \\
& $\mathrm{n}$ & $1.9 \times 10^{12}$ & 0.834 & 2.10 & 21 \\
\hline
\end{tabular}




\section{Results and discussion}

\subsection{Optimization of the sensor structure}

Certain non-linear effects are expected for the sensor output signal due to its operation mode. In Fig. 2 the responsivity as a function of the wavelength is displayed under no optical bias $\left(F_{\mathrm{L}}=0\right)$ and under uniform illumination $\left(530 \mathrm{~nm}, 2 \mathrm{~mW} \mathrm{~cm}{ }^{-2}\right)$, respectively. Data reveal that the responsivity when wide band gap doped layers are used is lower and it decreases significantly with the optical bias. Under optical bias conditions the band misalignment reduces the electrical field in the bulk and increases the recombination at the interfaces decreasing the carrier collection. The increased blue collection in dark observed in sensor \#M007192 is due to the higher front optical gap when compared with the other two sensors. Under different bias conditions $\left(530 \mathrm{~nm} ; 0<\Phi_{\mathrm{L}}<5 \mathrm{~mW} \mathrm{~cm}^{-2}\right.$ ) and in short circuit mode we have measured the light-to-dark signal ratio $\left[i_{\text {ac }}\left(\Phi_{\mathrm{L}}\right) / i_{\text {ac }}(0)\right]$ and the corresponding depletion widths [11].

In Fig. 3 we plot $S$ as a function of the applied optical bias, $\Phi_{\mathrm{L}}$. The depletion width dependence for sensor \#M007192 is superimposed. Results show that also the light-to-dark signal ratio depends strongly on the material of the doped layers. In the heterostructures the responsivity for low fluxes is high while in the homostructure only a small signal could be detected in the flux range analyzed. When the sensor has both doped layers based on a-SiC:H layers (\#M007192) the signal ratio steeply decreases. If only one layer is based on aSiC:H (\#M006301) the signal ratio also decrease but in a slower rate. Finally, in the homostructure (\#M006291) the sensor remains "blind" and only at higher light fluxes the signal ratio gently decreases.

The shrink of the depletion region and local quench of the electrical field at the illuminated regions increases with the presence of wide band/low conductive doped layers. This blocks the carrier collection at the illuminated interfaces (see Fig. 1) and decreases the light-to-dark signal ratio. In Fig. 4 the image intensity and the gray representation of a

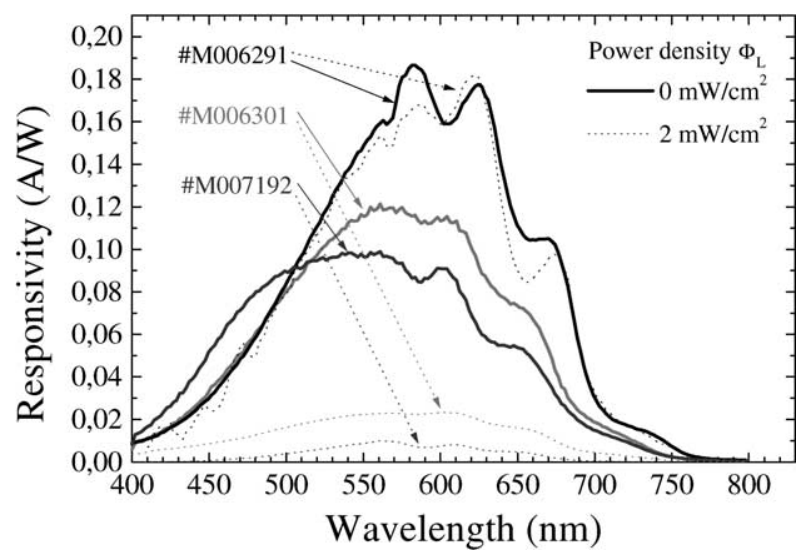

Fig. 2. Spectral sensitivity with (dot) and without (solid) applied optical bias.

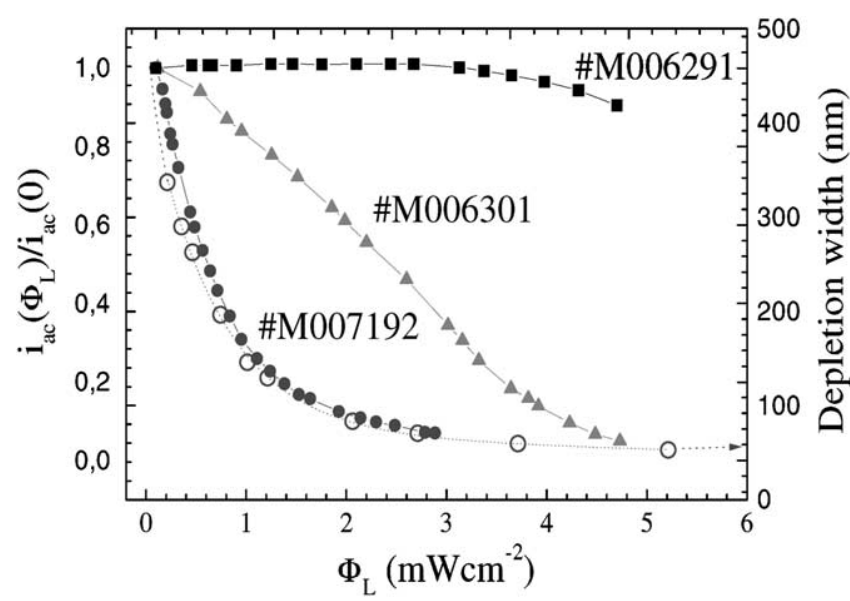

Fig. 3. Depletion width (open) and $i_{\mathrm{ac}}\left(\Phi_{\mathrm{L}}\right) / i_{\mathrm{ac}}(0)$ ratio (solid) dependence with $\Phi_{\mathrm{L}}$.

circle with $6 \mathrm{~mm}$ diameter halved by a $2 \mathrm{~mm}$ gap is displayed for two transducers having different doped n-layer conductivities and optical gaps. $\Phi_{\mathrm{L}}$ was kept constant at $5 \mathrm{~mW} \mathrm{~cm}^{-2}$ and the scanner intensity was about 20 times lower.

Results show that the observed differences in the resolution depend mainly on n-layer characteristics. The image signal and the spatial resolution are increased when the n-layer optical gap increases and the conductivity decreases. With high-conductive/low-band gap doped layers the carriers generated by the scanner inside the depletion regions (high potential barriers, dark regions) can drift along the doped layers towards the illuminated region (lower potential barriers) and are not collected leading to a lower image signal and to some blur. However, if highly resistive and wide-gap doped layers are used, the lateral flow of the carriers along the doped layers is minimized and the collection and/or recombination occurs beneath the generation point (position 2, Fig. 1) resulting in an increased spatial resolution. A resolution less than $20 \mu \mathrm{m}$ was measured in the sensor with both a-SiC:H low doped layers.

\subsection{Optimization of the read-out parameters}

Depending on the required application the sensor performance can be improved by a correct choice of the read-out parameters, like scanner beam spot size, scanner beam intensity and sensor electrical bias. Fig. 5 presents the normalized signal obtained on a sensor illuminated with a $5 \mathrm{~mm}$ strip using two different scanning beam diameters, only one scan is depicted. As one can see the increase in the diameter leads to a less steep variation of the signal when the scanner crosses the transition region between the illuminated and the dark zone. This fact leads to a blurring effect when the image is obtained as a gray level picture, but as no effect for black and white pictures if the threshold level is chosen at half the signal variation. Increasing the beam diameter leads to a fast acquisition time but limits the gray 

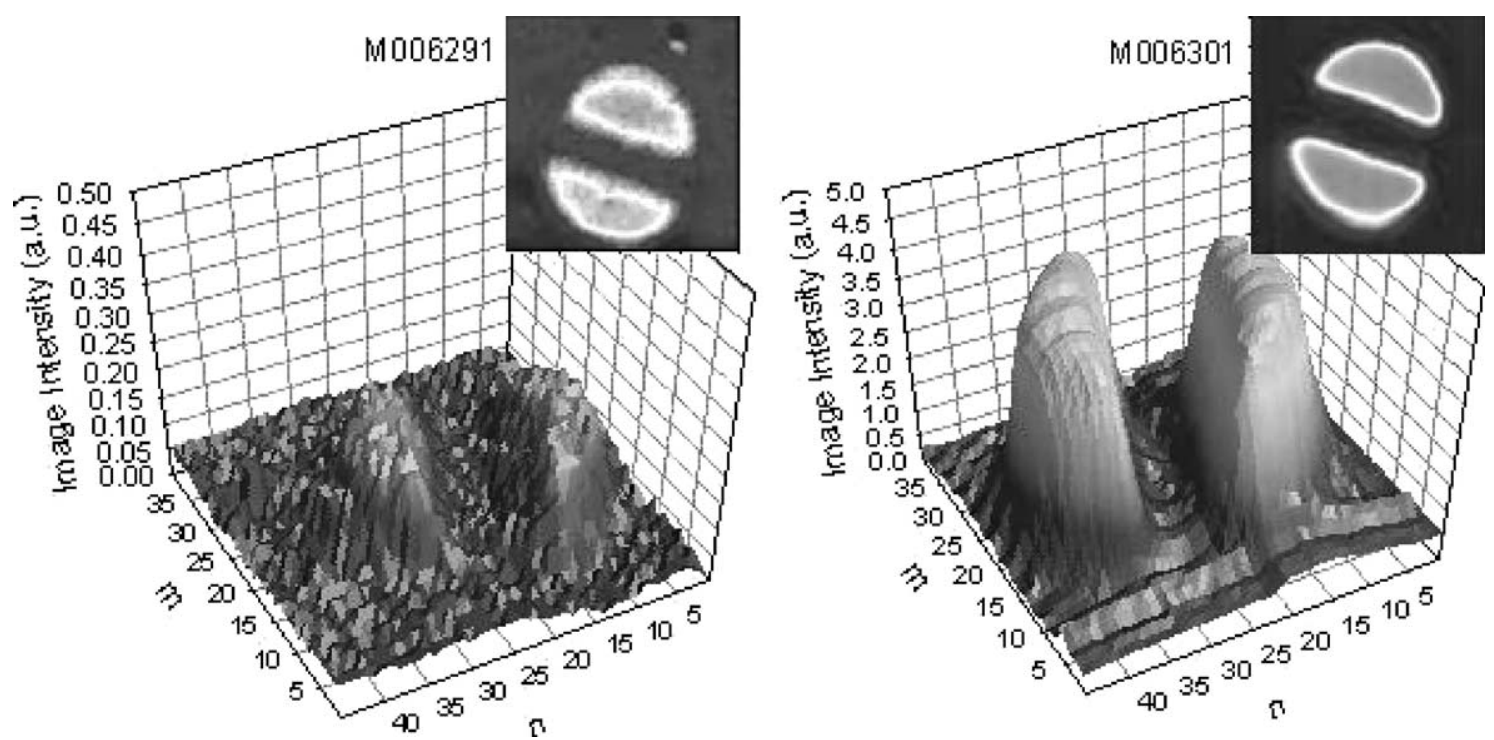

Fig. 4. Image signal and gray level image representation with sensors having different doped n-layer optical gaps.

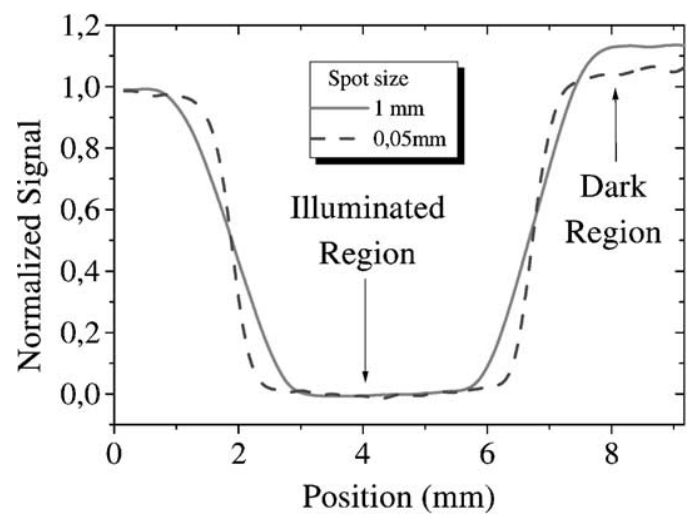

Fig. 5. Normalized signal for one-dimensional scan with different spot sizes.

level representation of the acquired images. So a trade-off between acquisition time and resolution is needed for a specific application.

In order to analyze the behavior of the sensor under different electrical and image brightness a pattern composed by dark and illuminated regions was projected on the active area of the sensor (\#M007192). Fig. 6a shows, at different image intensities, the dark-to-light variation of the output signal $\left(\Delta=i_{\mathrm{ac}}(0)-i_{\mathrm{ac}}\left(\Phi_{\mathrm{L}}\right)\right)$ as a function of the applied electrical bias. In Fig. $6 \mathrm{~b}$ the scans of the image under forward, reverse and zero bias voltage are represented. The image brightness $\left(2 \mathrm{~mW} \mathrm{~cm}{ }^{-2}\right)$, scanner intensity (two order of magnitude lower) and spot diameter $(50 \mu \mathrm{m})$ were kept constant.

Results show that the signal difference between dark and illuminated regions depends on the light flux and has a maximum at a voltage that depends on $\Phi_{\mathrm{L}}$. $\Delta$ is the maximum shifts from reverse to forward voltages as $\Phi_{\mathrm{L}}$ decreases. This effect is related with electrical field quench under illumination (see Section 2) that reduces locally the collection of the carriers generated by the scanner at the bright zones. At high $\Phi_{\mathrm{L}}$ the output signal saturates easily due to the shrink of the depletion region so by reverse bias the sensor the flux range before the saturation regime $\left(i_{\mathrm{ac}}\left(\Phi_{\mathrm{L}}\right) \sim 0\right)$ is enhanced and the collection in the dark
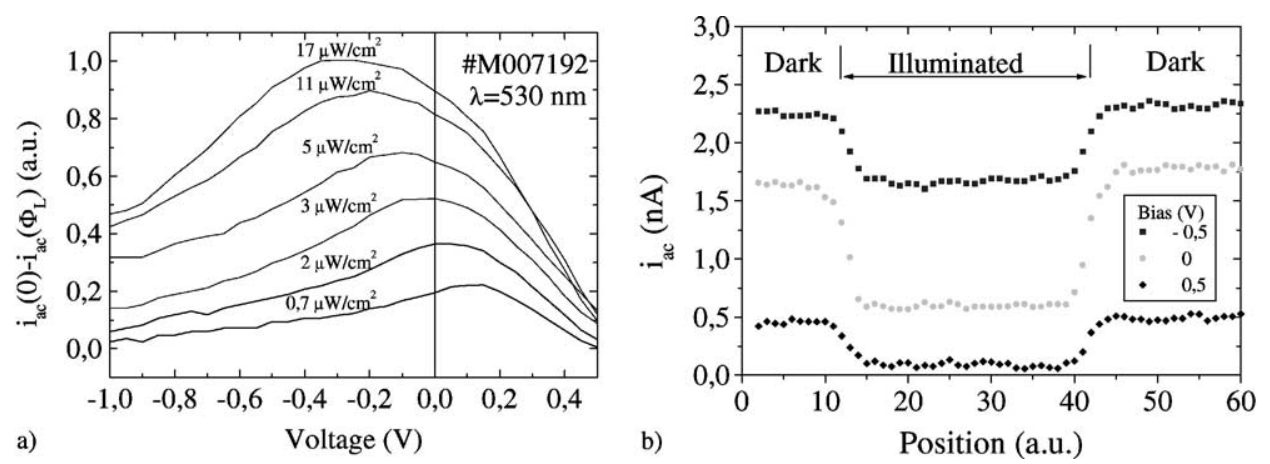

Fig. 6. (a) $\Delta=i_{\mathrm{ac}}(0)-i_{\mathrm{ac}}\left(\Phi_{\mathrm{L}}\right)$ as a function of the applied bias for different image intensities. (b) The ac photocurrent for one-dimensional scan under forward, reverse and zero bias. 


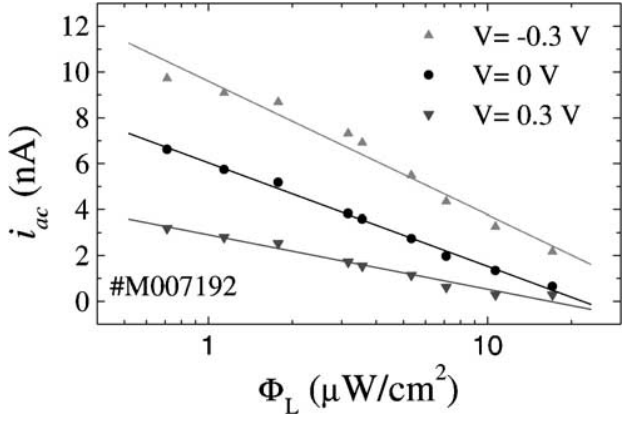

Fig. 7. The ac component of the photocurrent as a function of $\Phi_{\mathrm{L}}$ under reverse, forward and zero bias.

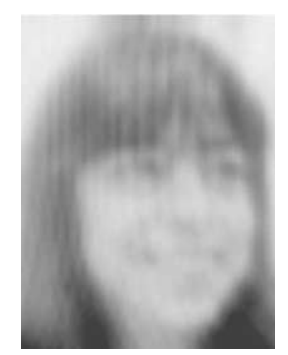

Fig. 8. \#M007192 LSP grayscale image.

regions improved (high $i_{\text {ac }}(0)$ ). At low $\Phi_{\mathrm{L}}$ the saturation regime is difficult to reach so by slightly forward biasing the sensor the collection of the carriers at the illuminated region will be lowered (low $i_{\mathrm{ac}}\left(\Phi_{\mathrm{L}}\right)$ ) while the collection at the dark zones remains almost the same. In both cases $\Delta$ will be enhanced. This trade-off between the electrical bias and the image brightness leads us to the conclusion that the best gray levels representations are achieved when the sensors operates in short circuit mode.

To analyze the sensor linearity and the effect of the image brightness on the LSP output signal in Fig. 7 we present the decrease in the output signal as a function of the light flux under different applied voltages. Data shows that sensor response is logarithmic, just like the human eye, independently of the applied voltage. Under reverse bias the image responsivity and the dynamic range are higher than in short circuit or forward bias. For the sensor \#M007192 and in reverse mode a dynamic range of two orders of magnitude with a responsivity of $6 \mathrm{~mA} \mathrm{~W}^{-1}$ and a sensitivity of $17 \mu \mathrm{W} \mathrm{cm} \mathrm{cm}^{-2}$ were achieved. As a possible application in Fig. 8 we display the grayscale photo representation obtained with the transducer \#M007192 in short circuit mode. No image processing algorithms were used.

\section{Conclusions}

Tailored $\mathrm{ZnO}: \mathrm{Al} / \mathrm{a}-\mathrm{pin} \mathrm{SiC}: \mathrm{H} / \mathrm{Al}$ imagers that uses a lowpower scanning beam to read-out the photogenerated carriers were presented. The sensor configuration and read-out parameters were optimized. The output characteristics were limited by the cell configuration and by the light source flux used to map the image onto the sensor. In the heterostructures with wide band gap/low conductivity doped layers an increase on the image signal optimized to the blue is achieved with a dynamic range of two orders of magnitude, a responsivity of $6 \mathrm{~mA} \mathrm{~W}^{-1}$ and a sensitivity of $17 \mu \mathrm{W}$ $\mathrm{cm}^{-2}$. Data reveals that the performance of an optimized sensor is enhanced by a tight control of the signal acquisition technique and read-out parameters such as scanner light source, mechanical system and bias conditions.

Further work has to be done in order to improve the competitivity of these kinds of sensors. Examples of possible applications are imaging and display devices, optical character recognition, facsimile transmitters, biometric readers and optical input point of sale terminals.

\section{Acknowledgements}

We would like to thank IPE for helpful discussions concerning this study. This work has been financially supported by PRAXIS/P/EEI/12183/1998, and by INIDA projects.

\section{References}

[1] J.W. Horton, R.V. Mazza, H. Dym, The scanistor-a solid state image sensor, Proc. IEEE 52 (1964) 1513-1519.

[2] P.W. Fry, Silicon photodiode arrays, J. Phys. E: Scientific Instrum. 8 (1975) 337-349.

[3] G.F. Amelio, Charge coupled devices, Scientific Am. 230 (1974) 22 31.

[4] D. Steven, H.W. Wilson, A $256 \times 256$ CMOS imaging array with wide dynamic range pixels and column-parallel digital output, IEEE J. Solid-State Circuits 33 (12) (1998) 2081-2083.

[5] H.K. Burke, G.J. Michon, Charge injection imaging: operating techniques and performance characteristics, IEEE Trans. Electron Dev. ED 23 (1976) 189-195.

[6] M. Vieira, M. Fernandes, J. Martins, P. Louro, A. Maçarico, R. Schwarz, M. Schubert, Improved resolution in a p-i-n image sensor by changing the structure of the doped layers, in: Proceedings of the Material Research Society Symposium, San Francisco, USA, In Amorphous and Heterogeneous Silicon thin Films, Vol. 609 (2000) A $32.45 / 281$.

[7] J.T. Wallmark, A new semiconductor photocell using lateral photoeffect, Proc. IRE 43 (1956) 474-483.

[8] J. Martins, M. Fernandes, P. Louro, A. Maçarico, M. Vieira, The contact geometry in a $2 \mathrm{D} \mu \mathrm{c}-\mathrm{Si}: \mathrm{H} \mathrm{P}-\mathrm{I}-\mathrm{N}$ imager flat-panel displays and sensors-principles, materials and processes, in: Proceedings of the Material Research Society Symposium, Vol. 558, San Francisco, USA, 1999, pp. 237-242.

[9] F. Sousa, J. Martins, M. Fernandes, A. Maçarico, R. Schwarz, M. Vieira, Image processing in a $\mu \mathrm{c}-\mathrm{Si}: \mathrm{H}$ p-i-n transducer, J. NonCrystalline Solids 266-269 (2000) 1228-1232.

[10] S.M. Sze, Physics of the Semiconductor Devices, 2nd Edition, Wiley, New York, 1981

[11] P. Louro, M. Vieira Yu. Vygranenko, M. Fernandes, R. Schwarz, M. Schubert, Bias dependent photocurrent collection in $\mathrm{p}-\mathrm{i}-\mathrm{n}$ a-Si:H/ $\mathrm{SiC}: \mathrm{H}$ heterojunction, Sens. Actuators A, 2001, in press.

[12] C. Koch, M. Ito, M. Schubert, J.H. Werner, Low-Temperature deposition of amorphous silicon based solar cells, Mat. Res. Soc. Symp. Proc. 575 (1999) 749-755. 


\section{Biographies}

Manuela Vieira was born in Lisbon, Portugal in 1951. She graduated in physics by the Faculty of Science of the University of Lisbon in 1974. In 1986 received the MSc in solid state physics-microelectronic by the New University of Lisbon. At that time she became Auxiliary Professor of semiconductor and microelectronics in ISEL, Lisbon where she is now Coordinator Professor in semiconductor/electronics and President of the Department of Electronics and Communications. In 1984 she became member of Center of Excellence for Microelectronics and Optoelectronics Processes-UNINOVA, Portugal. In 1993 she received the $\mathrm{PhD}$ in semiconductor materials from the New University of Lisbon. Currently she is the head of the group in applied research in microelectronic optoelectronic and sensors-GIAMOS/ISEL in Lisbon, Portugal. Dr. M. Vieira has several scientific papers and more than 12 years of experience in the field of thin films and devices, her research activities have been mainly related to the transient analysis and characterization of the transport properties of the semiconductor materials and device characterization.

Miguel Fernandes was born in Portugal in 1970. In 1993 he became researcher in the Center of Excellence for Microelectronics and Optoelectronics Processes-UNINOVA, Lisbon, Portugal. He graduated in physics and materials engineering by the Faculty of Sciences and Technology from the New University of Lisbon in 1995. Currently he is Assistant Professor in Electronics Department of ISEL, Lisbon, Portugal and investigator in the group of applied research in microelectronic optoelectronic and sensors (GIAMOS) in the same institution.

Yuriy Vygranenko was born in Ukraine in 1963. He received the degree in physics by the Chernovtsy University, Ukraine in 1985. Between 1985 and 1987 he worked as engineer at the optoelectronics device plant "Quartz", Ukraine. Later he became scientific collaborator in the Chernovtsy Department of the Institute of Materials Science Problems, where his research work was related to the physics and technology of the IV-VI narrow-gap semiconductors. In 1997 he received his $\mathrm{PhD}$ in physics from the Chernovtsy University. Currently he works as researcher in ISEL, Lisbon, Portugal.
Paula Louro was born in Portugal in 1967. In 1990 she became researcher in EID (a company of research and development in the field of electronics), Lisbon, Portugal, in the Department of Optoelectronics. She graduated in physics by the Faculty of Sciences from the University of Lisbon in 1990. In 1995 she received the MSc in material engineering by the New University of Lisbon.

Currently she is Assistant Professor in the Electronics Department of ISEL, Lisbon, Portugal, where she teaches electronic and semiconductor physics. Her main research interest is in the field of amorphous semiconductor thin films.

Reinhard Schwarz was born in Großßkööris, Germany in 1950 . He received the degree in physics and mathematics by the University of Stuttgart, Germany in 1977. He joined the Department of Physics in Neuchââtel University, Switzerland where he was teacher and where he received his $\mathrm{PhD}$ in 1982. After this date he became scientific collaborator in the Institute of Microtechnology, University of Neuchââtel, Switzerland until 1983 when he joined the group of Prof. S. Wagner in the Department of Electrical Engineering, Princeton University, USA. Later he joined the Physics Department E16 of Technische Universitäät Müünchen, Germany until 1996. Since then he teaches as invited professor in the Physics Department in Instituto Superior Téécnico, Lisbon, Portugal and coordinator professor in Electronics Department of Instituto Superior de Engenharia de Lisboa, Portugal.

Markus Schubert was born in Germany in 1958. He got the Diploma in electrical engineering by the Faculty of Electrical Engineering of the University of Stuttgart in 1985. The same year he joined the Institute of Physical Electronics in the University of Stuttgart as a research associate in the amorphous silicon group. The main working topic was on materials research for thin film solar cells. In 1992 he received the $\mathrm{PhD}$ degree in electrical engineering by the Faculty of Electrical Engineering of the University of Stuttgart. From 1992 to 1996 he taught in the University of Ulm (Germany) as professor of energy conversion and storage. Since 1996 he is group leader in "thin film sensors" at Institute of Physical Electronics, Stuttgart University, managing various research programs on thin film sensors and solar cells. Currently he is Associate Director of Institute of Physical Electronics at Stuttgart University (Germany). 